\title{
El efecto del rol docente en la presencia del pensamiento crítico de los foros en línea
}

\author{
Coordinadora del Programa de Producción Electrónica Multimedia de la
} Universidad Estatal a Distancia; isalas@uned.ac.cr

Recibido: 25 de enero de 2013

\section{RESUMEN}

Este artículo presenta los resultados de una investigación realizada para la disertación aplicada presentada ante Abraham S. Fischer School of Education de Nova Southeastern University y su objetivo es determinar si existe una correlación entre el rol docente y la presencia del pensamiento crítico en las argumentaciones de los estudiantes en los foros de discusión. A partir del análisis de contenidos se clasificaron 382 extractos de los mensajes de los estudiantes y 67 extractos de los mensajes del profesor. El análisis de los mensajes de los estudiantes se fundamentó en el modelo bidimensional de Garrison, Anderson y Archer (2001) y los indicadores del pensamiento crítico de Ennis (1993). La categorización de los mensajes del profesor se basó en los roles que este puede asumir en entornos virtuales de aprendizaje, según la revisión de literatura. La investigación responde a tres preguntas: a) ¿con qué frecuencia hay presencia del pensamiento crítico en las argumentaciones de los foros en línea?; b) ¿cuál es el rol que asume el profesor al participar en los foros de discusión en línea? y c) ¿cuál es el efecto del rol docente sobre el desarrollo del pensamiento crítico en las participaciones de los estudiantes de posgrado en los foros de discusión en línea? En promedio, solo el $20 \%$ de los mensajes de los estudiantes de posgrado se ubican en la fase superior del modelo bidimensional de Garrison y Anderson, Resolución; siendo la fase predominante la más inductiva, Activación. Pese a los bajos resultados en la fase de Resolución, el principal rol que asume el profesor es el Pedagógico. A partir de un análisis cualitativo se determinó que la correlación entre el rol docente y la presencia del pensamiento crítico es positiva. Finalmente, se sugiere que para futuras investigaciones se analice: el efecto que tiene el factor tiempo, el sentido de comunidad y el rol que asumen los estudiantes dentro de un grupo, en la presencia del pensamiento crítico en los foros de discusión.

\section{PALABRAS CLAVE}

Foros de discusión pensamiento crítico, análisis de contenido, cursos en línea, métodos de enseñanza.
Aceptado: 20 de febrero de 2013

\section{SUMMARY}

This paper presents the results of an investigation submitted to Abraham S. Fischer School of Education at Nova Southeastern University, and aims to determine whether there is a correlation between the teaching role and the presence of critical thinking in the arguments of students in the discussion forums. From content analysis 382 extracts were classified messages from students and 67 extracts the messages from the teacher. The analysis of the messages of the students was based on the two-dimensional model of Garrison, Anderson and Archer (2001) and Ennis'critical thinking indicators (1993). The message categorization was based on teacher's roles can assume that this virtual learning environments, according to the literature review. The research addresses three questions: a) how often critical thinking is present in the arguments of the online forums? b) What is the role assumed by the professor to participate in online discussion forums? c) What is the effect of the teaching role on the development of critical thinking in the units of graduate students in the online discussion forums? On average, only $20 \%$ of the messages of the graduate students are located in the upper phase bidimensional model of Garrison and Anderson, Resolution, being the most predominant phase inductive Activation. Despite the poor results in the resolution phase, the main role is assumed by the teacher pedagogy. From a qualitative analysis it was determined that the positive correlation between the teaching role and the presence of critical thinking. Finally, we suggest that future research is analyzed: the effect of the time factor, the sense of community and the role they assume students in a group, in the presence of critical thinking in discussion forums.

\section{KEY WORDS}

Online forums, critical thinking, content analysis, online courses, teaching methods. 


\section{INTRODUCCIÓN}

Desde el año 1999, la Universidad Estatal a Distancia en Costa Rica implementó el uso de plataformas para la administración y gestión de cursos en línea, Learning Management System (LMS). A partir de esta fecha, y de manera significativa, la oferta de cursos híbridos, bimodales y virtuales se ha incrementado. Según el Programa de Aprendizaje en Línea (2001, 2007, 2009), en el año 2001 se ofreció 45 cursos con componentes parcial o totalmente virtuales; para el año 2007, la oferta se extendió a 461 y, en el 2009, ascendió a 924. Actualmente, solo en el primero y segundo cuatrimestre del 2012 se registran más de 700 cursos haciendo prever que este año se superarán los 1000 cursos.

De manera paralela al crecimiento de la oferta académica en línea, las autoridades de la Universidad han implementado nuevas políticas, que aseguren la calidad y pertinencia de la oferta académica en línea. El Plan Académico 2008-2011 (UNED, 2008) impone el principio de formación, en el cual se detalla que es necesario potenciar en los estudiantes la habilidad de discriminar la información y de tomar decisiones partiendo del pensamiento crítico.

Por su parte, el Modelo Pedagógico de la Universidad exhorta a la academia para que, a partir del uso de los recursos tecnológicos, se propicie en el estudiante el desarrollo de habilidades de pensamiento y de resolución de problemas. Este documento especificaque el modelo de comunicación didáctica debe considerar: "Incorporar actividades que exijan del alumno procesos de pensamiento crítico, reflexivo y creativo" (UNED, 2004, p. 47).

Sin embargo, la experiencia de la autora del presente estudio, como investigadora y productora de cursos en línea, coincide con las investigaciones realizadas por Cabero, (2000), García, Ruiz y Domínguez (2007), Garrison y Cleveland-Innes (2005), entre otros, quienes indican que no siempre se logra este objetivo. Una interrogante transciende en el quehacer universitario: ¿en qué medida los cursos en línea están propiciando el desarrollo del pensamiento de alto nivel?

La comunicación didáctica, a la que se refiere el Modelo Pedagógico (UNED, 2004), es un elemento que puede provocar en los estudiantes la motivación y el compromiso en los procesos de aprendizaje, brindando oportunidades y retos, guiándolos a procesos de pensamiento de nivel superior. Esta labor es competencia del profesor del curso (Bender, 2003; Salmon, 2003).

Bender (2003) asegura que es el profesor la persona responsable de minimizar la distancia transaccional en la relación profesor-estudiantecontenidos. A través de una efectiva instrucción en línea, su accionar es definitorio en los resultados obtenidos. Garrison y Anderson atribuyen a la labor del profesor una función integradora al "facilitar el pensamiento crítico y los resultados de alto nivel en el contexto del e-learning" (2005 P.97) y, al igual que Bender, opinan que es el profesor quien debe "garantizar el equilibrio transaccional adecuado" (2003, p. 96).

Por las razones anteriores, el tema de esta investigación se centró en la interrogante sobre el papel de la mediación del profesor y el rol que éste asume en un foro de discusión en línea, como un factor determinante de la presencia de habilidades de pensamiento crítico en las argumentaciones de los estudiantes, a nivel de posgrado.

\section{- Antecedentes y justificación}

La evidencia contenida en las investigaciones relacionadas demuestra que los estudiantes que usan los foros de discusión en línea, para publicar argumentos de índole académica, no logran llegar a niveles de pensamiento de orden superior y que los foros muchas veces se convierten en espacios de interacción social más que cognitiva (Cabero, 2000; García, Ruiz \& Domínguez, 2007; Garrison \& Cleveland-Innes, 2005; Melaré, 2007; Monke, 2005; Tirado, 2002). 
El estudio realizado por Gamboa y Salas (2009) revela que en la universidad involucrada en esta investigación es necesario no solo capacitar a los profesores para el desarrollo de competencias tecnológicas, sino también en la comunicación didáctica en entornos virtuales. Tal y como lo argumentan Bautista, Borges y Forés (2008) y García et al. (2007) no basta con conocer el potencial tecnológico que se posee, se hace necesaria una mediación capaz de llevar a los estudiantes en un proceso continuo de construcción del conocimiento y del pensamiento de alto nivel. En palabras de Duart y Sangrà se trata de: "crear espacios tecnológicamente potentes para después usarlos pedagógicamente...la tecnología tiene que ser un recurso al servicio del proceso de aprendizaje" (2000, p. 17). Esto solo es posible con una propuesta metodológica y una mediación docente que sean capaces de crear ambientes de aprendizaje en los que se potencie el pensamiento de alto nivel (Garrison \& Cleveland-Innes, 2005).

Si se toma como referencia la investigación de Salmon (2003), en la que analizó más de 3000 mensajes en foros durante 2 años, la evidencia apunta que las interacciones que se desarrollan en los foros de discusión, no logran ubicarse en la fase de construcción del conocimiento y de desarrollo. Esta fase se caracteriza por promover la metacognición, la exploración del propio pensamiento y el control sobre sus procesos de conocimiento.

Garrison y Anderson (2005), Garrison y Cleveland-Innes (2005), Oh y Jonassen (2007) exponen que una de las causas atribuidas al problema expuesto es el poco aprovechamiento del potencial implícito para la argumentación encontrado en el uso de las herramientas de comunicación, dada su misma asincronía, lo cual permite el tiempo de reflexión y análisis a los estudiantes.

Al señalar de manera específica la labor del profesor, los resultados obtenidos en la investigación de Gamboa y Salas indican que "los profesores parecen saber utilizar las herramientas, por ejemplo los foros y blogs, pero no tienen aún las habilidades para ser orientadores, guías, facilitadores de los procesos en línea" (2009, p. 17). En este mismo estudio, los estudiantes manifestaron "no percibir que se logren niveles de análisis, síntesis y reflexión en estas actividades y que además es notoria la ausencia del profesor en los procesos" (2009, p. 17).

Lo anterior señala que, más allá del poco aprovechamiento del potencial de comunicación, es necesario reconocer cómo la metodología utilizada por los profesores determina los niveles de implicación y desarrollo de los estudiantes (García, Márquez, Bustos, Miranda \& Espíndola, 2008; Garrison \& Anderson, 2005; Garrison \& Cleveland-Innes, 2005; Melaré, 2007; Salmon, 2002).

\section{- Marco teórico}

\section{Definición de pensamiento crítico}

Norris y Ennis definen el pensamiento crítico como el "pensamiento razonable y reflexivo que está enfocado hacia la toma de decisiones sobre en qué creer y qué hacer" $(1989$, p. 3). Al realizar un análisis de esta definición, Bruning, Schraw y Ronning (2007) exponen que el concepto reflexivo debe ser entendido como la comprensión de la naturaleza del objeto de estudio y no como la solución de problemas. Además, exponen que el término enfocado, al que se refieren Norris y Ennis (1989), implica que se centra en la mejor comprensión del objeto de estudio y no en el simple pensamiento. A esto hay que sumar que "el contenido del pensamiento crítico, a diferencia de la solución de problemas, suele ser una creencia o un motivo que deseamos examinar detalladamente" (Bruning, Schraw \& Ronning, 2007, p.259), con el fin de lograr la toma de decisiones fundamentadas.

El potencial del desarrollo del pensamiento reflexivo en los estudiantes radica, según Oh y Jonassen (2007), en hacer visible el pensamiento, 
en explicitar las creencias, las concepciones, los conocimientos, y en argumentar y refutar.

No es posible generar pensamiento crítico sin conocimiento $y$, al mismo tiempo, el último es el resultante del pensamiento, ya que es el que permite evaluar y juzgar la información que se recibe (Bruning et al., 2007; Perkins, 1997). Bruning et al. son enfáticos: "El conocimiento suministra la base para juzgar la credibilidad de la información o de los puntos de vista nuevos y nos ayuda a examinar de modo crítico nuestras metas y objetivos" (2007, p. 260). Por esta razón, el individuo debe desarrollar una serie de tácticas o estrategias mentales, que utiliza con el fin de que el proceso cognitivo se torne más comprensible y factible.

Las estrategias o tácticas cognitivas tienen como principal función establecer los caminos a seguir en la resolución de dilemas. Por ejemplo, la inferencia ha demostrado ser una de las estrategias de pensamiento más eficientes para comprender las situaciones, evaluarlas y juzgarlas: "consiste en establecer una conexión entre dos o más unidades de conocimiento" (Bruning et al., 2007, p. 261). Hay dos tipos de inferencia: la deducción y la inducción. Ambas funciones cognitivas son producto del pensamiento formal, estos procesos son considerados subconjuntos del pensamiento (Crespo, 2006; Mayer, 1986).

\section{Descriptores del pensamiento reflexivo y crítico}

Para pensar de manera razonable y reflexiva, Ennis (1993) propone que las personas deben lograr dos clases principales de actividades de pensamiento crítico: las disposiciones y las habilidades o capacidades.

Las disposiciones son los rasgos afectivos tendientes a favorecer el pensamiento crítico; a esto Norris y Ennis (1989) le llaman el espíritu crítico. Son disposiciones, según Bruning, Schraw y Ronning: "la apertura mental, el intento de estar bien informado y la sensibilidad hacia las creencias, los sentimientos y el conocimiento ajenos" (2007, p. 260). Las disposiciones propias del espíritu crítico expuestas por Ennis (1993) son: a) juzgar la credibilidad de las fuentes; b) identificar las conclusiones, las razones, y las hipótesis; c) juzgar la calidad de un argumento, incluida la aceptabilidad de sus razones, las hipótesis y las pruebas; d) elaborar y defender una posición sobre un tema; e) realizar, de manera acertada, preguntas con el fin de aclarar dudas; f) planificar experimentos y juzgar los diseños experimentales; g) definir los términos de una forma apropiada para el contexto; h) tener la mente abierta; i) estar bien informados; j) sacar conclusiones cuando se justifique, pero con cautela.

Por otra parte, las habilidades son "las capacidades cognitivas para pensar de modo crítico, como centrarse, analizar y juzgar" (Bruning et al., 2007, p. 260). La taxonomía de Ennis (Norris \& Ennis, 1989) declara las habilidades o capacidades del pensamiento crítico que se describen según las siguientes clasificaciones:

1. Elemental: a) focalizarse en la pregunta, b) analizar los argumentos, c) formular y responder preguntas clarificadoras.

2. Soporte básico: a) juzgar la credibilidad de la fuente, b) hacer y juzgar observaciones.

3. Inferencia: a) hacer y juzgar deducciones, b) hacer y juzgar inducciones, c) hacer y juzgar juicios de valor.

4. Avanzada: a) definir términos y juzgar definiciones, b) identificar los supuestos.

5. Estrategias y tácticas: a) decidir una acción a seguir, b) interactuar con otros.

Esta lista interdependiente de disposiciones y habilidades puede, según Ennis (1993), proveer una guía para evaluar el pensamiento crítico. La lista puede guiar un conjunto de metas que se propongan dentro de un plan de estudios, o puede ser la base para una tabla de especificaciones de lo que es pensar críticamente. 


\section{Presencia cognitiva}

La presencia cognitiva es definida como el espacio intelectual que favorece el desarrollo y potenciación del discurso crítico sostenido. Su objetivo es propiciar la adquisición y aplicación del conocimiento de alto nivel; es decir, el análisis, la construcción, el aprendizaje significativo y la comprensión, a partir de la reflexión y el discurso (Garrison \& Anderson, 2005).

El pensamiento crítico tiene valor práctico siempre y cuando se profundice en la comprensión de las experiencias y en sus significados. La importancia del pensamiento crítico reside en la autenticación del conocimiento $y_{1}$ a la vez, en la generación de nuevo conocimiento; de ahí la importancia de generar procesos de metacognición en el estudiante. (Garrison \& Anderson, 2005).

Para Garrison, Anderson y Archer (2001), el pensamiento crítico debe concebirse como un proceso y un resultado. Como resultado, es el mejor entendimiento de una perspectiva individual de los hechos, a partir del profundo y significativo entendimiento del objeto de estudio, al hacer uso de las disposiciones y capacidades desarrolladas. Sin embargo, evaluar el pensamiento crítico como un producto presenta la dificultad de un complejo proceso cognitivo. Por esa razón, como proceso, supone la adquisición de habilidades de pensamiento que ayudarán en gran medida a su comprensión. Este proceso puede ser apoyado por herramientas, externas al sujeto, que permitan evaluar el discurso crítico y la reflexión. En otras palabras, será necesario realizar un entramado entre el mundo privado, el individual, que es reflexivo y da significado a los objetos de estudio y el mundo social, público, que es cooperativo y da soporte a la presencia cognitiva.

En el Modelo Bidimensional de Investigación Práctica presentado por Garrison, Anderson y Archer, se integra el mundo privado y públi$\mathrm{CO}$, donde el estudiante es el eje central para la creación de procesos que garanticen la presencia cognitiva. El modelo describe cuatro fases en el proceso de desarrollo de la presencia cognitiva en contextos de e-learning, una advertencia que hacen los investigadores es que estas fases no son inalterables, sino que pueden ser "telescopadas o invertidas en la medida en que se considere o no la visión y la comprensión" (2001, p.88), obsérvese la Figura 1.

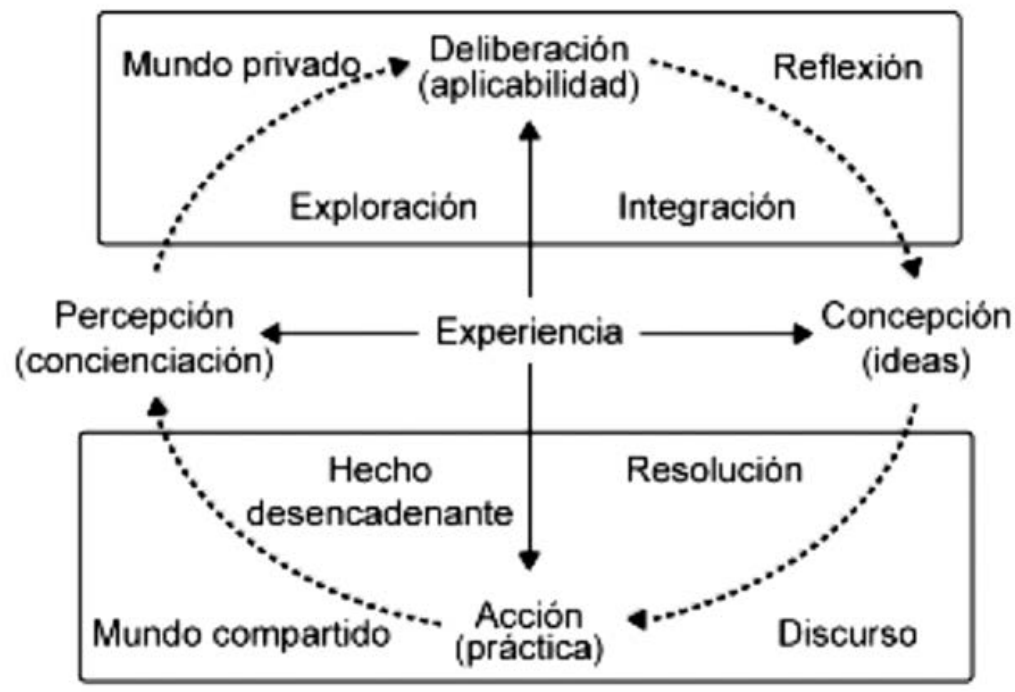

Figura 1. Modelo Bidimensional de Investigación Práctica. (De El e-learning en el siglo XXI. Investigación y práctica (p. 89), por D. Garrison y T. Anderson, 2005, Barcelona, España: Ediciones Octaedro, S.L.) 
Las cuatro fases del modelo se describen a continuación (Garrison \& Anderson, 2005):

\section{Activación o hecho desencadenante:} esta fase implica la presentación de un hecho o actividad donde los estudiantes se impliquen. Los procesos aquí observados se describen como evocativos e inductivos.

2. Exploración: esta etapa exige entender la naturaleza del problema, conocer los elementos que la componen, buscar la información más relevante y buscar posibles explicaciones. Al finalizar esta fase, los estudiantes deben reconocer aquello que es relevante en su tema u objeto de estudio a partir de un proceso inquisitivo y divergente.

3. Integración: su objetivo es la construcción del significado. Los aspectos propios de la sistematización de ideas son resultados aquí, así como su integración. Es una fase reflexiva, pero exige también el discurso crítico con el fin de forjar la comprensión. Esta fase es la más difícil de detectar desde la enseñanza y la investigación, ya que la evidencia de la integración de las ideas y la construcción de significados debe ser inferida desde la comunicación dentro de la comunidad que se ha creado. Se describe como un proceso tentativo-convergente.

4. Resolución: esta fase consiste en plantear una alternativa de solución al dilema o al problema; por lo tanto, requiere de la prueba deductiva de la solución. El razonamiento deductivo incluye una inferencia lógica en la que se deduce y se generaliza a partir de los conocimientos y la información explícita. Esto puede hacerse de dos maneras: a) reduciendo la complejidad mediante un marco de significado o de explicación, o b) descubriendo una solución contextual específica. El proceso de resolución se caracteriza por ser comprometido y deductivo.

\section{Presencia y rol docente}

El Modelo Bidimensional de Investigación Práctica de Garrison et al. (2001) establece que todo el potencial del e-learning debe ser planificado y mediatizado por un profesor competente, capaz de evaluar lo que ofrece la tecnología para diseñar ambientes de aprendizajes interactivos y basados en la investigación. La presencia docente ha sido definida como "el diseño, facilitación y orientación de los procesos cognitivo y social con el objetivo de obtener resultados educativos significativos desde el punto de vista personal y docente" (Garrison et al., 2001, p. 98).

Por otra parte, Anderson, Rourke, Garrison y Archer (2001) advierten que esta función se genera previamente al inicio del curso, en los procesos de planificación y diseño, y se extiende a lo largo del proceso al facilitar el discurso y al intervenir directamente cuando se considere pertinente. En esta labor, el profesor asume diferentes roles o cargos. Para efectos prácticos, y como base para evaluar el actuar de los profesores en los entornos virtuales para la enseñanza y el aprendizaje, pueden resumirse tres roles del profesor: el organizativo, el social y el pedagógico (Garrison \& Anderson, 2005; Gisbert, 2002; Cabero \& Román, 2006).

Para Garrison y Anderson (2005), Cabero y Román (2006), García y sus colegas (2007), el rol organizativo implica que el profesor asume un papel de planificador y coordinador de la logística de un curso. Esto es, define los contenidos así como los objetivos, planifica las estrategias didácticas y evaluativas, diseña y elabora materiales o los selecciona, define los tiempos de acción, entre otras acciones.

El profesor que asume un rol social tiene como objetivo crear, en el contexto de enseñanza y aprendizaje, una comunidad, caracterizada por la colaboración, la confianza, el respeto, la libertad de expresión y la no discriminación (Bender, 2003; Cabero \& Román, 2006; García et al., 2007; Garrison \& Anderson, 2005; Garrison \& Vaughan, 2008; Salmon, 2004). 
En el rol pedagógico, el profesor debe demostrar que es competente en su área de especialidad y que sabe cómo interceder entre los estudiantes y los contenidos, cómo poner en práctica las estrategias didácticas y evaluativas que se han planificado para el curso, y cómo usar acertadamente los recursos (Garrison \& Anderson, 2005; Cabero \& Román, 2006; García et al., 2007; Gisbert, 2005). En este rol, el profesor debe comprometer y activar la participación de los estudiantes, haciendo que los procesos de aprendizaje se tornen significativos y relevantes (Bender, 2003).

\section{METODOLOGÍA}

El enfoque metodológico utilizado en este estudio fue el análisis cuantitativo de contenidos. Este es un estudio predominantemente descriptivo. La confiabilidad se determinó realizando el cálculo intercodificadores (Hernández, Fernández y Baptista, 2006), incluyendo dos codificadores adicionales a la investigadora para poder obtener el grado de consenso entre parejas.

La muestra fue elegida de manera no probabilística y por conveniencia, incluyó dos grupos en diferentes períodos académicos. El curso seleccionando correspondió a uno ofertado en el nivel de maestría en la Universidad Estatal a Distancia de Costa Rica. Los foros pertenecieron a la oferta 2009 y 2010 de este curso.

La unidad de análisis de este estudio fueron los mensajes extraídos de los foros, tanto de los estudiantes como de los profesores. La revisión de contenidos se realizó con información archivada "ex post facto". El Cuadro 1 muestra la cantidad de extractos de textos publicados y revisados. Los mensajes fueron segmentados para poder obtener el mismo número de codificaciones a realizar, tanto por la investigadora como por los codificadores asistentes.

\section{Instrumentos}

Para evaluar las transcripciones de los mensajes contenidos en los foros se diseñaron hojas de codificación de contenidos. Según lo indican Hernández et al. (2006), estas permiten estudiar los procesos de comunicación objetiva y sistemáticamente, al cuantificar y clasificar los contenidos, convirtiéndolos en elementos posibles de describir y evaluar. La objetividad en la construcción de los instrumentos se buscó a partir de la definición de categorías, subcategorías y criterios.

Las categorías que se usaron para clasificar los mensajes de los estudiantes se desprendieron del Modelo Bidimensional de Investigación Práctica presentado por Garrison, Anderson y Archer (2001). Las subcategorías se definieron usando la Taxonomía de Ennis (Norris \& Ennis, 1989), que detalla los procesos socio-cognitivos evidenciados en el pensamiento crítico.

Cada subcategoría contiene tópicos del pensamiento crítico descritos en la Taxonomía de Ennis (Norris \& Ennis, 1989, Ennis, 1993), que permitieron desagregar los procesos sociocognitivos expresados, con el fin de observar las destrezas de pensamiento asociadas.

Cuadro 1. Número de mensajes y extractos de texto de los foros del módulo 5 en cada año seleccionado

\begin{tabular}{|c|c|c|c|}
\hline Año & $\begin{array}{c}\text { Número de } \\
\text { mensajes }\end{array}$ & $\begin{array}{c}\text { Número de extractos de } \\
\text { textos de los estudiantes }\end{array}$ & $\begin{array}{c}\text { Número de extractos de } \\
\text { textos del Profesor }\end{array}$ \\
\hline 2009 & 117 & 194 & 33 \\
\hline 2010 & 108 & 188 & 34 \\
\hline Totales & 225 & 382 & 67 \\
\hline
\end{tabular}


En el caso de la codificación de los mensajes del profesor, los roles representan las categorías que ellos pueden asumir y los indicadores son funciones relacionadas a cada rol. Para determinar los roles y sus funciones, se tomó como referencia la decisión de la investigadora de resumir los roles del profesor en tres: el organizativo, el social y el pedagógico. De igual forma, se aplicaron las funciones que se describen en cada uno de estos roles y se ubicaron en el instrumento como indicadores.

Los códigos descritos en las Hojas de Codificación de Contenidos de los Mensajes de los Estudiantes y de los Profesores fueron colocados en el paquete de software Atlas.ti@ 6.1 de Scientific Software. Esta aplicación sirvió como herramienta de apoyo al permitir el análisis cualitativo de los mensajes. A través de la creación de Unidades Hermenéuticas en Atlas. ti $\odot$, se hizo posible tener en una sola pantalla del paquete: los extractos textuales de los foros, los códigos y sus indicadores.

\section{RESULTADOS}

\section{- Mensajes de los estudiantes}

El Cuadro 2 desglosa los resultados obtenidos por la investigadora al codificar los argumentos de los estudiantes de los años 2009 y 2010.
Un total de $67,3 \%$ de los mensajes se ubicaron en la categoría Activación, seguido de la categoría Integración con un 20,4\%; luego se ubica Resolución con el 8,9\%, y Exploración con el 1,8\%.

Véase en el Cuadro 3 que, en la categoría Activación, la subcategoría que predomina es Analiza argumentos, con un total del $64,4 \%$, y el indicador con más porcentaje es Identifica argumentos válidos, que obtiene un $38,7 \%$, seguido por Identifica las conclusiones, con un total del $14,7 \%$.

El Cuadro 4 muestra la categoría Integración, en ella la subcategoría Hacer y juzgar deducciones es la que obtiene el mayor porcentaje, un $16,8 \%$, resultado de la suma de los indicadores: A partir de la información, Derivando conclusiones e Interpretando las declaraciones, las dobles negaciones, las condiciones necesarias.

Para la categoría Resolución, solo es distinguible la subcategoría Interactúa con otros, que muestra un $5,5 \%$ del total asignado, distribuido entre los indicadores Presentar una posición a una audiencia en particular y Usar estrategias retóricas (véase Cuadro 5).

En el caso de la categoría Exploración, en la subcategoría Juzga la credibilidad de la fuente con un total de $1,8 \%$ de las codificaciones, los únicos indicadores que reflejaron puntaje por parte de la investigadora fueron: Acuerdo con otras fuentes y Conflicto de interés (véase Cuadro 6).

Cuadro 2. Resumen por categorías de las codificaciones de la investigadora en los foros de los estudiantes, para los años 2009 y 2010.

\begin{tabular}{|l|c|c|c|c|}
\hline \multicolumn{1}{|c|}{ Categorias } & $\mathbf{2 0 0 9}$ & $\mathbf{2 0 1 0}$ & Total & $\%$ \\
\hline Activación & 127 & 130 & 257 & 67,3 \\
\hline Exploración & 3 & 4 & 7 & 1,8 \\
\hline Integración & 43 & 35 & 78 & 20,4 \\
\hline Resolución & 17 & 17 & 34 & 8,9 \\
\hline No aplica en ninguna categoría del pensamiento crítico & 4 & 2 & 6 & 1,6 \\
\hline Totales & 194 & 188 & 382 & 100,0 \\
\hline
\end{tabular}


Cuadro 3. Detalle de las codificaciones de la investigadora en los foros de los estudiantes en los años 2009 y 2010, para la categoría Activación

\begin{tabular}{|l|l|c|c|c|c|}
\hline \multicolumn{1}{|c|}{ Subcategoria } & \multicolumn{1}{|c|}{ Indicadores } & 2009 & $\mathbf{2 0 1 0}$ & Total & $\%$ \\
\hline $\begin{array}{l}\text { Focaliza la } \\
\text { pregunta }\end{array}$ & $\begin{array}{l}\text { Identifica o formula criterios para } \\
\text { juzgar posibles respuestas }\end{array}$ & 2 & 3 & 5 & 1,3 \\
\hline \multirow{4}{*}{$\begin{array}{l}\text { Analiza } \\
\text { argumentos }\end{array}$} & Identifica argumentos válidos & 71 & 77 & 148 & 38,7 \\
\cline { 2 - 6 } & Identifica las conclusiones & 26 & 30 & 56 & 14,7 \\
\cline { 2 - 6 } & Observa similitudes y diferencias & 8 & 8 & 16 & 4,2 \\
\cline { 2 - 6 } & Resume y sintetiza & 16 & 10 & 26 & 6,8 \\
\hline \multirow{3}{*}{$\begin{array}{l}\text { Responde y hace } \\
\text { preguntas para } \\
\text { clarificar }\end{array}$} & ¿Podría decir algo más acerca de eso...? & 1 & 0 & 1 & 0,3 \\
\cline { 2 - 6 } & ¿Qué diferencia hace? & 2 & 1 & 3 & 0,8 \\
\cline { 2 - 6 } & ¿Qué entiende usted por...? & 1 & 0 & 1 & 0,3 \\
\cline { 2 - 6 } & $\begin{array}{l}\text { ¿Cómo funciona ese caso, parece estar ofrecien- } \\
\text { do una excepción que se aplica a esta situación? }\end{array}$ & 0 & 1 & 1 & 0,3 \\
\hline Total Activación & & 127 & 130 & 257 & 67,3 \\
\hline
\end{tabular}

Cuadro 4. Detalle de las codificaciones de la investigadora en los foros de los estudiantes en los años 2009 y 2010, para la categoría Integración

\begin{tabular}{|c|c|c|c|c|c|}
\hline Subcategoría & Indicadores & 2009 & 2010 & Total & $\%$ \\
\hline \multirow{3}{*}{$\begin{array}{l}\text { Hacer y juzgar } \\
\text { deducciones }\end{array}$} & A partir de la información & 18 & 9 & 27 & 7,1 \\
\hline & Derivando conclusiones & 15 & 11 & 26 & 6,8 \\
\hline & $\begin{array}{l}\text { Interpretando las declaraciones, las dobles } \\
\text { negaciones, las condiciones necesarias }\end{array}$ & 7 & 4 & 11 & 2,9 \\
\hline \multirow{3}{*}{$\begin{array}{l}\text { Hacer y juzgar } \\
\text { inducciones }\end{array}$} & $\begin{array}{l}\text { Generalizando, referidas a instancias típicas, } \\
\text { limitaciones de cobertura }\end{array}$ & 1 & 3 & 4 & 1,0 \\
\hline & $\begin{array}{l}\text { Explicando y haciendo hipótesis, criterios para } \\
\text { explicar la evidencia, tener coherencia, predecir }\end{array}$ & 1 & 3 & 4 & 1,0 \\
\hline & $\begin{array}{l}\text { Investigando, observando evidencia } \\
\text { y contraevidencia }\end{array}$ & o & 1 & 1 & 0,3 \\
\hline \multirow{2}{*}{$\begin{array}{l}\text { Hacer y juzgar los } \\
\text { juicios de valor en } \\
\text { consideración a la: }\end{array}$} & $\begin{array}{l}\text { Dependencia en el más alto orden de } \\
\text { los valores y principios }\end{array}$ & o & 3 & 3 & 0,8 \\
\hline & Pertinencia de los hechos & 1 & 1 & 2 & 0,5 \\
\hline Total integración & & 43 & 35 & 78 & 20,4 \\
\hline
\end{tabular}


Cuadro 5. Detalle de las codificaciones de la investigadora en los foros de los estudiantes, en los años 2009 y 2010, para la categoría Resolución

\begin{tabular}{|c|c|c|c|c|c|}
\hline Subcategoría & Indicadores & 2009 & 2010 & Total & $\%$ \\
\hline $\begin{array}{l}\text { Definir términos y } \\
\text { juzgar definiciones }\end{array}$ & $\begin{array}{l}\text { Por sinónimo, dando ejemplos y no ejemplos, o por } \\
\text { el uso de expresión equivalente... }\end{array}$ & 2 & 1 & 3 & 0,8 \\
\hline \multirow{2}{*}{$\begin{array}{l}\text { Identificar } \\
\text { asunciones }\end{array}$} & Asunciones y supuestos no explícitos & o & 1 & 1 & 0,3 \\
\hline & Supuestos necesarios & 3 & 1 & 4 & 1,0 \\
\hline \multirow{3}{*}{$\begin{array}{l}\text { Decidir en una } \\
\text { acción }\end{array}$} & Define un problema & 1 & o & 1 & 0,3 \\
\hline & $\begin{array}{l}\text { Examinar teniendo en cuenta la situación en su } \\
\text { totalidad y decidir }\end{array}$ & o & 1 & 1 & 0,3 \\
\hline & Seleccionar criterios para juzgar soluciones & 1 & 2 & 3 & 0,8 \\
\hline \multirow{2}{*}{$\begin{array}{l}\text { Interactuar con } \\
\text { otros }\end{array}$} & $\begin{array}{l}\text { Presentar una posición a una } \\
\text { audiencia en particular }\end{array}$ & 10 & 10 & 20 & 5,2 \\
\hline & Usar estrategias retóricas & o & 1 & 1 & 0,3 \\
\hline Total Resolución & & 17 & 17 & 34 & 8,9 \\
\hline
\end{tabular}

Cuadro 6. Detalle de las codificaciones de la investigadora en los foros de los estudiantes, en los años 2009 y 2010, para la categoría Exploración.

\begin{tabular}{|l|l|c|c|c|c|}
\hline \multicolumn{1}{|c|}{ Subcategoría } & \multicolumn{1}{|c|}{ Indicadores } & $\mathbf{2 0 0 9}$ & $\mathbf{2 0 1 0}$ & Total & \% \\
\hline \multirow{2}{*}{$\begin{array}{l}\text { Juzgar la credibilidad de la fuente } \\
\text { con criterios de: }\end{array}$} & Acuerdo con otras fuentes & 3 & 0 & 3 & 0,8 \\
\cline { 2 - 7 } & Conflicto de interés & 0 & 4 & 4 & 1,0 \\
\hline Total Exploración & 3 & 4 & 7 & 1,8 \\
\hline
\end{tabular}

Las decisiones de los codificadores en la asignación de categorías a los extractos de texto de los estudiantes se observan en el Cuadro 7. Se han unificado las decisiones de ambos codificadores para los foros de los años 2009 y 2010. La categoría Activación predomina con un $59,6 \%$. Dentro de esta, la subcategoría Analiza argumentos fue la que reunió la mayor parte de las codificaciones, un total de $55,7 \%$. El indicador Identifica argumentos válidos sobresalió con un $35,9 \%$, seguido de los indicadores Resume y sintetiza con un $9 \%$ e Identifica las conclusiones con un $8,1 \%$.
La categoría que sigue en predominancia es Integración, con un total de $23 \%$. Dentro de ella, se destacó la subcategoría Hacer y juzgar deducciones, que conglomeró el 19,2\% de las codificaciones y el indicador $A$ partir de la información, que mostró un $9,8 \%$ del total, seguido por Derivando conclusiones con el $8,1 \%$.

El criterio de los codificadores señala que la categoría Resolución se hace evidente en el $14,8 \%$ de los extractos de texto. Dentro de esta categoría, la subcategoría Definir términos y juzgar definiciones abarcó el $8,1 \%$ del total de las codificaciones y dentro de esta, dos son los 
indicadores que se distinguieron: Por estrategias como la definición de actos, expresar una posición, formulando una pregunta apropiada, identificación de errores, con un $5,4 \%$ y Por sinónimo, dando ejemplos y no ejemplos, expresión equivalente con el $2,7 \%$.

La categoría que obtuvo el menor porcentaje de codificaciones asignadas por los codificadores es Exploración, solo obtuvo el 1,6\% del total de las categorizaciones realizadas por los codificadores (véase Cuadro 7). Los indicadores que obtuvieron asignación de extractos de textos son: Acuerdo con otras fuentes, con un $1,4 \%$, y Conflicto de interés, al cual le fue asignado un $0,1 \%$ del total de las codificaciones.

Las diferencias porcentuales obtenidas entre los dos codificadores y la investigadora se señalan en el Cuadro 8. En ella se puede verificar que la categoría Activación supera las otras para los tres codificadores.

Tanto la investigadora como los dos codificadores coinciden en que la segunda categoría que se manifiesta con mayor frecuencia en los foros es Integración; Resolución es la categoría que ocupa el tercer lugar de manifestación en los foros; Exploración es la que tiene menor porcentaje de diferencia entre los tres codificadores.

Con el propósito de analizar esta distribución y ordenamiento porcentual de categorías, en el Cuadro 9 se han incluido las frecuencias acumuladas, absolutas y relativas. Puede observarse que solamente en la primera categoría, Activación, quedan clasificados más de la mitad de los mensajes y para la segunda categoría, Integración, el porcentaje acumulado alcanza el $85 \%$. Las otras dos categorías, Resolución

Cuadro 7. Resumen de las codificaciones de los codificadores 1 y 2 en los foros de los estudiantes, para los años 2009 y 2010.

\begin{tabular}{|l|c|c|c|c|}
\hline \multicolumn{1}{|c|}{ Categorías } & $\mathbf{2 0 0 9}$ & $\mathbf{2 0 1 0}$ & Total & $\%$ \\
\hline Activación & 241 & 214 & 455 & 59,6 \\
\hline Exploración & 9 & 3 & 12 & 1,6 \\
\hline Integración & 85 & 91 & 176 & 23,0 \\
\hline Resolución & 47 & 66 & 113 & 14,8 \\
\hline No aplica en ningún criterio del pensamiento crítico & 6 & 2 & 8 & 1,0 \\
\hline Totales & 388 & 376 & 764 & 100,0 \\
\hline
\end{tabular}

Cuadro 8. Diferencia porcentual entre las codificaciones asignadas por los dos codificadores y la investigadora para los argumentos de los estudiantes en los foros del 2009 y 2010

\begin{tabular}{|l|c|c|c|}
\hline \multicolumn{1}{|c|}{ Categorias } & \% Investigadora & \% Codificadores 1 y 2 & Diferencias \\
\hline Activación & 67,3 & 59,6 & 7,7 \\
\hline Exploración & 1,8 & 1,6 & 0,2 \\
\hline Integración & 20,4 & 23,0 & $-2,6$ \\
\hline Resolución & 8,9 & 14,8 & $-5,9$ \\
\hline No aplica & 1,6 & 1 & 0,6 \\
\hline
\end{tabular}


y Exploración, solamente abarcan el restante $15 \%$ de los mensajes.

A partir de estos datos se puede concluir que: a) la fase Activación predomina en los foros analizados; b) la fase de Exploración prácticamente es "saltada" dentro del proceso; c) la participación de los estudiantes presenta un incremento en la fase de Integración; y d) es evidente una disminución de las participaciones clasificadas en la fase de Resolución (véase Figura 2).

\section{Mensajes del profesor}

El Cuadro 10 muestra los resultados obtenidos en cada uno de los roles asumidos por el profesor en el año 2009 y 2010, según las decisiones de codificación tomadas por la investigadora. Aquellos indicadores en los cuales no se asignó codificación fueron omitidos.

El profesor tuvo 33 aportes en el foro en el año 2009, de estos 2 fueron clasificados de tipo

Cuadro 9. Distribución de los mensajes de los estudiantes clasificados según categoría, frecuencias simples y acumuladas $(\mathrm{N}=1132)$

\begin{tabular}{|l|c|c|c|c|c|c|}
\hline & Investigadora & Codificadores & \multicolumn{2}{|c|}{ Total } & Frecuencia & $\%$ \\
\hline Categoría & $\mathrm{N}^{\circ}$ & $\mathrm{N}^{\circ}$ & $\mathrm{N}^{\circ}$ & $\%$ & Acumulada & Acumulado \\
\hline Activación & 257 & 455 & 712 & 62,9 & 712 & 62,9 \\
\hline Integración & 78 & 176 & 254 & 22,4 & 966 & 85,3 \\
\hline Resolución & 34 & 113 & 147 & 13,0 & 1113 & 98,3 \\
\hline Exploración & 7 & 12 & 19 & 1,7 & 1132 & 100 \\
\hline No aplica & 6 & 8 & 14 & $*$ & $*$ & $*$ \\
\hline Total & 382 & 764 & 1146 & 100 & 1132 & 100 \\
\hline
\end{tabular}

*/Se consideran solo el porcentaje válido de casos (excluyendo la categoría no aplica).

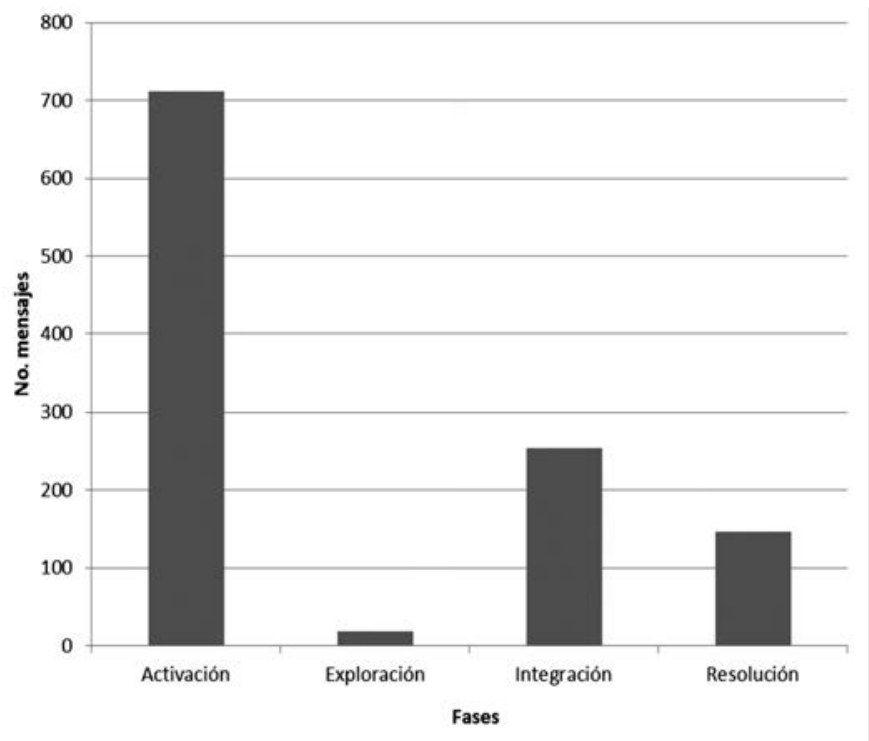

Figura 2. Distribución de los mensajes de los estudiantes según fase ( $N=1132)$. 
Cuadro 10. Indicadores que reflejan frecuencias en las publicaciones del profesor dentro de los foros del 2009 y 2010 , según la investigadora $(N=67)$

\begin{tabular}{|c|c|c|c|c|c|}
\hline Rol & Indicador & 2009 & 2010 & Total & $\%$ \\
\hline \multirow{2}{*}{ Organizativo } & $\begin{array}{l}\text { Plantea observaciones, en el foro, de manera general so- } \\
\text { bre el contenido del curso }\end{array}$ & 1 & 1 & 2 & 3,0 \\
\hline & $\begin{array}{l}\text { Diseña métodos, determina cuáles serán las actividades } \\
\text { que se llevarán a cabo }\end{array}$ & 1 & 3 & 4 & 6,0 \\
\hline \multicolumn{2}{|c|}{ Total rol organizativo } & 2 & 4 & 6 & 9,0 \\
\hline \multirow[b]{2}{*}{ Social } & Busca establecer áreas de consenso & 3 & o & 3 & 4,5 \\
\hline & $\begin{array}{l}\text { Estimula y motiva a los estudiantes en el valor de sus } \\
\text { participaciones para el crecimiento grupal }\end{array}$ & 4 & 6 & 10 & 14,9 \\
\hline \multicolumn{2}{|l|}{ Total rol social } & 7 & 6 & 13 & 19,4 \\
\hline \multirow{8}{*}{ Pedagógico } & $\begin{array}{l}\text { Inyecta conocimiento desde diferentes fuentes, por } \\
\text { ejemplo libros de texto, artículos, Internet, experiencias } \\
\text { personales }\end{array}$ & o & 1 & 1 & 1,5 \\
\hline & $\begin{array}{l}\text { Abre espacios de comunicación continua para la tutoría } \\
\text { permanente, atendiendo las dudas y las necesidades es- } \\
\text { pecíficas de los estudiantes }\end{array}$ & 1 & 1 & 2 & 3,0 \\
\hline & $\begin{array}{l}\text { Asegura el acompañamiento, al motivar y guiar los pro- } \\
\text { cesos de aprendizaje reconociendo los estilos particula- } \\
\text { res de cada estudiante }\end{array}$ & 1 & 0 & 1 & 1,5 \\
\hline & Diagnostica los errores de concepto & 2 & 1 & 3 & 4,5 \\
\hline & $\begin{array}{l}\text { Estimula en el estudiante el desarrollo de las destrezas } \\
\text { para la autorregulación y autogestión de los procesos de } \\
\text { aprendizaje }\end{array}$ & 1 & 2 & 3 & 4,5 \\
\hline & $\begin{array}{l}\text { Estimula la discusión y el análisis de las situaciones, a } \\
\text { partir de la argumentación reflexiva }\end{array}$ & 13 & 7 & 20 & 29,8 \\
\hline & $\begin{array}{l}\text { Orienta las discusiones y establece conclusiones a partir } \\
\text { de ellas }\end{array}$ & 1 & 9 & 10 & 14,9 \\
\hline & $\begin{array}{l}\text { Promueve la interacción entre los participantes y con los } \\
\text { contenidos }\end{array}$ & 5 & 3 & 8 & 11,9 \\
\hline \multicolumn{2}{|c|}{ Total rol pedagógico } & 24 & 24 & 48 & 71,6 \\
\hline \multicolumn{2}{|c|}{ Totales } & 33 & 34 & 67 & 100,0 \\
\hline
\end{tabular}


Organizativo lo que significa un $6,1 \% ; 24$ de las participaciones tuvieron características del rol Pedagógico, lo que representa el $72,7 \%$ y el $21,2 \%$ de las publicaciones fueron clasificadas dentro del rol Social.

En el año 2010, el número de publicaciones del profesor fue de 34 . Un 11,8\% de ellas fueron categorizadas en el rol Organizativo. En el $70,6 \%$ de las publicaciones, o sea en 24 de ellas, el profesor asumió un rol Pedagógico y el 17,6\% de las veces asumió un rol Social.

En el caso del rol Organizativo, dos son las categorías que son evidentes al analizar los mensajes del profesor dentro del foro: a) Plantea observaciones, en el foro, de manera general sobre el contenido del curso y b) Diseña métodos, determina cuáles serán las actividades que se llevarán a cabo.

En el rol Social,los mensajes categorizados son identificados con dos indicadores: a) Busca establecer áreas de consenso y b) Estimula y motiva a los estudiantes en el valor de sus participaciones para el crecimiento grupal.

El rol Pedagógico es el que predomina en los argumentos del profesory en él tres son los indicadores con mayor porcentaje: a) Estimula la discusión y el análisis de las situaciones; a partir de la argumentación reflexiva; b) Orienta las discusiones y establece conclusiones a partir de ellas y c) Promueve la interacción entre los participantes y con los contenidos.
Con el fin de verificar los resultados anteriores con las clasificaciones realizadas por los codificadores, se procedió a unir las codificaciones de ambos para revisar las diferencias con la investigadora. Obsérvese en el Cuadro 11 que los codificadores atribuyen el 91,8\% de los mensajes al rol Pedagógico del profesor, mientras que la investigadora le atribuye a este rol el $71,6 \%$ del total de los mensajes; esto denota una diferencia de $20,2 \%$.

La segunda diferencia entre los codificadores y la investigadora se muestra en el rol Social. Los codificadores indican que un $5,2 \%$ de los mensajes tienen características de este rol, mientras que la investigadora indica un 19,4\%: la diferencia de criterios es de $14,2 \%$. Por último, la investigadora categoriza un $6 \%$ más que los codificadores a los mensajes de tipo Organizativo. Ambos codificadores señalan que $3 \%$ de los mensajes publicados por el profesor se clasifican en este rol. Mientras que la investigadora atribuyó este rol al 9\% de los mensajes.

Al considerar que la investigadora y los codificadores siguieron las mismas pautas para codificar el rol que asume el profesor en el foro, es pertinente comprobar una posible asociación significativa entre las frecuencias obtenidas en cada caso. Para ello, se elaboró una tabla de contingencia y la hipótesis fue contrastada por medio del estadístico Chi-cuadrado de Pearson (véase el Cuadro 12).

Cuadro 11. Diferencia porcentual de los roles asumidos por el profesor en los foros del 2009 y 2010 , entre la investigadora y los codificadores

\begin{tabular}{|l|c|c|c|}
\hline \multirow{2}{*}{ Rol } & Investigadora & Codificadores & Diferencias \\
\cline { 2 - 4 } & $\%$ & $\%$ & $\%$ \\
\hline Organizativo & 9,0 & 3,0 & 6,0 \\
\hline Social & 19,4 & 5,2 & 14,2 \\
\hline Pedagógico & 71,6 & 91,8 & $-20,2$ \\
\hline
\end{tabular}


El valor obtenido para el estadístico Chi-cuadrado tiene una probabilidad inferior al 1\%; por lo tanto, se concluye que la codificación por roles no es independiente de quienes la realizan, sea la investigadora o los codificadores.

Específicamente, hay diferencias importantes, a un nivel de significancia del $1 \%$, en la codificación del rol Pedagógico y del Social, las marcas obtenidas en cada uno de estos roles fueron de $-0,201493$ y de 0,141791, respectivamente. No se presentó diferencia significativa para el rol Organizativo, al cual corresponden la menor cantidad de las frecuencias y la diferencia que marcó fue del 0,059701. Lo anterior se verificó por medio de una prueba de diferencia entre las correspondientes proporciones, la cual se muestra en el Cuadro 13.

Los indicadores que predominan dentro del rol Pedagógico, según los codificadores son: a) Asegura el acompañamiento, al motivar y guiar los procesos de aprendizaje reconociendo los estilos particulares de cada estudiante, al cual se le atribuye el $23,1 \%$ del total de los mensajes; b) Promueve la interacción entre los participantes y con los contenidos, con un 22,4\%; c) Estimula la discusión y el análisis de las situaciones; a partir de la argumentación reflexiva, con un $17,2 \%$ del total de mensajes y d) Orienta las discusiones y establece conclusiones a partir de ellas, con el $11,2 \%$.

Cuadro 12. Distribución de los mensajes del profesor codificados según rol por parte de la investigadora y los codificadores y prueba de asociación

\begin{tabular}{|l|c|c|c|}
\hline \multicolumn{1}{|c|}{ Rol } & Investigadora & Codificadores & Total \\
\hline Pedagógico & 48 & 123 & 171 \\
\hline Social & 13 & 7 & 20 \\
\hline Organizativo & 6 & 4 & 10 \\
\hline Total & 67 & 134 & 201 \\
\hline
\end{tabular}

\begin{tabular}{|c|c|c|c|c|}
\hline \multicolumn{2}{|c|}{ Estadístico } & Valor & gl & p \\
\hline Chi Cuadrado & Pearson & 14,36 & 2 & $0,0008 *$ \\
\hline
\end{tabular}

$\mathrm{p}=$ probabilidad $; \mathrm{gl}=$ grados de libertad .

*Diferencia significativa (probabilidad menor a 1\%).

Cuadro 13. Prueba de diferencias entre proporciones para la codificación de roles que asume el profesor

\begin{tabular}{|l|c|c|}
\hline \multicolumn{1}{|c|}{ Rol } & Diferencia & Probabilidad \\
\hline Pedagógico & $-0,201493$ & $0,00008 *$ \\
\hline Social & 0,141791 & $0,00250^{*}$ \\
\hline Organizativo & 0,059701 & 0,08682 \\
\hline
\end{tabular}

*Diferencia significativa al $1 \%$. 
En el rol social, para los codificadores, los indicadores predominantes fueron: a) Estimula el trabajo colaborativo y la socialización de la información, con un 2,2\% del total de los mensajes y b) Estimula y motiva a los estudiantes en el valor de sus participaciones para el crecimiento grupal, igualmente con un $2,2 \%$.

Es el criterio de los codificadores que el indicador predominante en el rol Organizativo es Diseña métodos, determina cuáles serán las actividades que se llevarán a cabo, al cual le atribuyeron 4 mensajes de texto, lo que representa el $2,2 \%$ del $3 \%$ total atribuido a este rol.

\section{- Análisis de los datos}

\section{Confiabilidad}

El cálculo de confiabilidad para las codificaciones de los mensajes de los estudiantes fue de 0,52 y para las codificaciones de los mensajes de los profesores fue de 0,71 . Dado que: 1) aún los investigadores en el campo del análisis de contenidos no tienen un "nivel convencional aceptable" (Garrison \& Anderson, 2005, p. 183) para el cálculo de confiabilidad; 2) los resultados de esta investigación no solo muestran las decisiones de codificación de la investigadora, sino también las de los codificadores; 3) el contenido codificado tiene un componente altamente filosófico, lo cual dificulta más llegar a acuerdos, y 4) los valores obtenidos en la confiabilidad superan el 0.5; es por eso consideran como representativos los resultados obtenidos, pues superan las posibilidades de ser producto de la casualidad.

Si bien el coeficiente de confiabilidad fue alto para las codificaciones de los mensajes de los profesores, un 0,76 , existieron algunas diferencias significativas en la forma que la investigadora identificó los roles Pedagógico y Social respecto de los codificadores. En particular, los codificadores clasificaron en mayor proporción los mensajes dentro del rol Pedagógico, lo que puede estar indicando varias causas: a) la necesidad de contar con criterios más precisos para distinguir entre las tres categorías; b) la de tener un marco de selección de categorías e indicadores más reducido, dado que la densidad de las selecciones se concentran más en algunos indicadores y reflejan mayores diferencias; c) la posibilidad de que existan mensajes con elementos referentes a más de un rol, es decir, mensajes que contemplen combinaciones de estos, lo cual dificulta más la toma de decisiones.

No obstante a pesar de que el valor del ChiCuadrado obtenido tiene una probabilidad inferior a $1 \%$, lo cual indica que las clasificaciones realizadas no pudieron ser independientes de los codificadores, es un hecho que el rol Pedagógico presentó una frecuencia evidentemente superior a los otros dos, lo que indica que la coincidencia entre codificadores y la investigadora reafirma su prevalencia.

\section{Análisis de los mensajes de los estudiantes}

Fase activación: como puede observarse en los resultados obtenidos, la fase Activación del modelo bidimensional de investigación práctica de Garrison et al. (2001) fue la que predominó en los argumentos de los estudiantes dentro de los foros analizados durante los años 2009 y 2010.

La fase predominante fue la más inductiva y restrictiva por naturaleza, ya que el profesor, por lo general, define los objetivos o se refiere a algún tema central, iniciando la conversación (Garrison \& Anderson, 2005). La sugerencia es que el profesor brinde un tema y las preguntas o problemas sean generadas por los estudiantes. Esta última situación no se evidenció; los estudiantes respondieron a preguntas o instrucciones de trabajo puntuales planteadas por el profesor, en las que se preguntó por uno o varios conceptos o por una definición.

Pudo verificarse que los estudiantes buscaron información, específicamente, referencias bibliográficas para publicar su participación en los foros. Si bien la respuesta fue válida, sobre el significado que se pidió, esta no se desprendió 
de un análisis profundo de una situación, sino de la identificación de un texto y de un autor que les sirvió de apoyo para responder.

Fase integración: esta ocupó el segundo lugar en predominancia y en ella el indicador con mayor puntaje fue Hacer y juzgar deducciones.

Garrison y Anderson exponen que esta fase "se orienta a la construcción de significados" (2005, p. 90) y se caracteriza por ser reflexiva y por buscar la comprensión, "es el proceso de elaborar una solución o explicación apropiada" (2005, p. 92). La didáctica implica, según estos autores, que el profesor exponga ideas a fin de que sean corroboradas por los estudiantes para provocar que se analicen los temas desde otras perspectivas y que expliquen experiencias. La investigación confirma que en esta fase el profesor logró realizar una de sus mejores intervenciones al plantear un dilema, ya que este tipo de mediaciones incitaron a los estudiantes a analizar la situación y luego posicionarse con un argumento a partir de la reflexión, el razonamiento y la deducción.

Fue posible verificar cómo los estudiantes lograron, tomando referencias de su contexto, analizar la situación, exponer sus argumentos. Esto es coherente con lo expuesto por Henning (2004) y Salmon (2002) sobre la importancia de la interpretación individual de los acontecimientos propios del contexto y la cultura para provocar la reflexión y la toma de posición sobre las ideas.

En las argumentaciones clasificadas en la fase Integración, se verificó que los estudiantes solo hacían citaciones textuales para sostener su argumento, no para usarlo como único recurso de su respuesta, sino como un apoyo. El planteamiento de un caso de la vida real provocó en los estudiantes llegar a la tercera fase del modelo bidimensional de investigación práctica. Tal como lo indican Garrison y Anderson (2005) y Garrison y Vaughan (2008) es el uso de estrategias que lleven a la investigación, al razonamiento de problemas y dilemas, a la contextualización práctica lo que puede provocar el avance entre una fase a otra del modelo.
Fase resolución: esta es la fase del modelo de Garrison et al. (2001) que ocupó el tercer lugar. Según Garrison y Anderson, esta fase es la que denota la resolución de un dilema o problema, pero inevitablemente los resultados obtenidos hacen que se planteen nuevas preguntas, "activando en nuevos ciclos de indagación y, por lo tanto, promoviendo el aprendizaje continuo" (2005, p. 90).

Importante aspecto en este momento es el avance hacia la clarificación, la evidencia de tomar una posición ante otros, la decisión de acción (Norris y Ennis, 1989). Por lo tanto, el reto es que los estudiantes demuestren el razonamiento sobre un problema, la solución directa o indirecta, la cual requerirá pruebas, comprobar y defender; es aquí donde el mundo privado y público convergen (Garrison \& Anderson, 2005). Pudo evidenciarse que pocas fueron las intervenciones de los estudiantes que lograron ubicarse en esta fase.

En la investigación Shea, Li y Pickett (2006), los estudiantes manifestaron que sentían que cuando el profesor se ocupaba de identificar áreas de acuerdo y desacuerdo, esto les ayudaba en la resolución de sus diferencias hasta alcanzar consensos y percibían que lograban niveles elevados de conexión y aprendizaje. Partiendo de estos hallazgos, podría deducirse que no se lograron tener mejores resultados en la fase de Resolución por ausencia de una mayor motivación e intervención del profesor. No hay evidencia en los foros analizados, para esta investigación, de que el profesor provocara el desequilibrio cognitivo a los estudiantes, confrontando posiciones para luego buscar consensos, dado que la mayoría de los mensajes se limitaron a contestar preguntas directas.

Tampoco hay evidencia en los foros de que el profesor o los mismos estudiantes evaluaran cualitativamente el discurso o la argumentación, recomendación que hace Garrison y Anderson (2005) para provocar una participación productiva hacia el pensamiento crítico. Todas las respuestas se dieron como válidas, lo cual 
indica poca interacción y poca reflexión acerca de la veracidad de las exposiciones.

Fase exploración: esta fase ocupó el último lugar de presencia en los foros analizados. Los dos indicadores que marcaron puntuaciones, tanto en las decisiones de la investigadora como las resoluciones de los codificadores, fueron Acuerdos con otras fuentes y Conflicto de interés.

La importancia de la fase Exploración radica en estimular el pensamiento divergente y en que los estudiantes se muestren inquisitivos al revisar el valor de la información que se posee. La ausencia de esta fase podría ser uno de los factores que expliquen la poca incidencia en la fase Resolución. Esto es, al no investigar y comprobar primero algunos argumentos, los estudiantes no tuvieron criterios claros para interactuar con otros, defender y comprobar posiciones, tal como lo indica Bruning et al. (2007): no habían bases para juzgar la credibilidad, dado que el conocimiento es la fuente para pensar críticamente.

Garrison y Anderson exponen que esta fase es la que "implica lanzar y presentar ideas para corroborar o para oponerse a otras, recibir nuevas perspectivas, explicar las experiencias y hacer observaciones y comentarios sobre el valor de la información" (2005, p. 92). Si esto no se hace y esta fase es saltada, como se ha demostrado, se puede explicar por qué la fase Resolución es pobre en frecuencias obtenidas, no hay conocimiento y por lo tanto tampoco argumentos para debatir.

Garrison y Anderson señalan que "la tendencia es resolver bien las dos primeras fases, la tercera no tanto y la cuarta, dejarla al margen" (2005, p. 93). El Cuadro 9 y la Figura 2, muestran que la primera fase, Activación, es resuelta de manera satisfactoria en esta investigación. Contrario a la experiencia de Garrison y Anderson (2005), la segunda fase, Exploración, quedó casi anulada. La tercera fase, Integración, volvió a elevar los resultados, quiere decir que se busca la solución, la convergencia de ideas, la síntesis, pero no de manera significativa ya que el porcentaje es bajo. Al igual que lo indican Garrison y Anderson (2005), la fase Resolución decayó significativamente, pese a ser la fase en la que se manifiesta el tipo de pensamiento comprometido, deductivo, en donde se defiende una idea, aspectos propios del pensamiento crítico.

\section{Análisis de los mensajes del profesor}

Rol pedagógico: la estadística de las frecuencias de los mensajes publicados por el profesor refleja que el rol predominante fue el Pedagógico. Los dos indicadores en los que coincidieron tanto la investigadora como los codificadores son: Estimula la discusión y el análisis de las situaciones a partir de la argumentación reflexiva y Promueve la interacción entre los participantes y con los contenidos.

El primer indicador es precisamente lo que Shea, Li y Pickett (2006) interpretan como una labor necesaria por parte del profesor para lograr niveles altos de pensamiento; sin embargo, el porcentaje de presencia de este indicador en el foro fue bajo.Hizo falta una mayor presencia de este indicador para lograr llegar a la fase Resolución.

En los extractos de texto analizados pudo observarse cómo el profesor logra realizar dos acciones importantes: a) estimular a los estudiantes por sus participaciones y b) provocar nuevas participaciones y reflexión en los estudiantes. Si bien la presencia de este tipo de mensaje es porcentualmente baja, es necesario preguntarse: ¿por qué a pesar de estas intervenciones del profesor, los estudiantes no lograron llegar en mayores porcentajes de participación a las fases de Integración y sobre todo a la de Resolución?

Para responder esta interrogante, dos hipótesis surgen: la primera es el tiempo de respuesta o intervención del profesor, la cual se caracterizó por tener de 3 a 4 accesos en los cuales publicaba de 5 a 9 mensajes seguidos, con preguntas, aclaraciones o felicitaciones por el trabajo realizado, 
pero luego se ausentaba por periodos de 3 a 5 días. En otras palabras, el lapso de tiempo dado entre la publicación del estudiante y la retroalimentación del profesor puede ser un factor condicionante de una mayor tasa de respuestas y argumentación por parte de los estudiantes.

La segunda hipótesis para intentar responder por qué a pesar del estímulo los estudiantes no logran mayor presencia cognitiva, versa sobre la poca atención individualizada que se brindó. En los textos analizados se evidenció que el profesor no cuestionó o retó a un estudiante en particular. Cuando el profesor se dirigió a alguien en particular fue porque esa persona le hizo una pregunta directa. En otras palabras, si un estudiante se mostró tímido en sus respuestas y no logró argumentar sólidamente su posición, el profesor no le pidió ampliar, mejorar, verificar, demostrar y tampoco lo hicieron sus compañeros, por lo que cualquier respuesta fue válida. Verificar estas dos hipótesis es un reto para una futura investigación.

Al confrontar lo sucedido y tratar de responder con la teoría, es posible identificar la importancia del rol del profesor en la distancia transaccional. Bender (2003) afirma que una mediación efectiva en línea, puede provocar la reducción de la distancia transaccional a partir de la atención individualizada, el reconocimiento de las necesidades de los estudiantes según sus estilos de aprendizaje y la construcción de andamios o puentes cognitivos para favorecer la construcción del conocimiento. Esta autora también argumenta que el estilo del profesor en sus intervenciones puede favorecer el compromiso, la motivación, la discusión y la argumentación.

De acuerdo con lo anterior, se concluye que el profesor no logró reducir la distancia transaccional de manera efectiva. No se estimuló en los estudiantes mayor compromiso y participación. El equilibrio transaccional que la presencia docente debe procurar, según Garrison y Anderson (2005), no logró el diálogo reflexivo que se esperaba en los estudiantes a nivel de posgrado.
Rol social: el rol Social ocupó el segundo lugar dentro de la presencia docente de los foros en línea. Al observar con detalle los valores porcentuales del rol social que el profesor asumió en los foros, puede determinarse que son bajos. Los resultados, tanto de la investigadora como de los codificadores, indican que no se estimuló el trabajo colaborativo, el buscar puntos de consenso y por ende el intercambio de información. Este aspecto pudo ser determinante en la presencia cognitiva, dado que es necesario crear comunidad e identidad, antes de pretender la construcción colectiva del conocimiento (Garrison \& Anderson, 2005; Salmon, 2004). La presencia social provee el ambiente necesario de colaboración para que los estudiantes se comprometan con alcanzar sus metas y generar un discurso cohesivo. Una futura investigación podría analizar los roles de los estudiantes en un entorno social y cómo estos pueden incidir en la presencia cognitiva.

Rol organizativo: es criterio de la investigadora y de los codificadores que el indicador predominante en el actuar del profesor en este rol fue Diseña métodos, determina cuáles serán las actividades que se llevarán a cabo.

Las acciones realizadas por el profesor demuestran que fue capaz de establecer "la estructura de la ejecución a desarrollar" (Cabero \& Román, 2006, p. 208), explicó las normas de funcionamiento de las actividades, estableció calendarios o tiempos de trabajo y pautas de conducta (Garrison \& Anderson, 2005).

\section{Efecto del rol docente sobre el desarrollo del pensamiento crítico en los foros de discusión en línea}

Para contestar la tercera pregunta de investigación: ¿cuál es el efecto del rol del docente sobre el desarrollo del pensamiento crítico en las participaciones de los estudiantes de posgrado, en los foros de discusión en línea?, se hizo un análisis cualitativo de los mensajes del profesor y de los estudiantes.

Se distingue en las intervenciones del profesor cómo se privilegió la formulación de preguntas 
directas, las cuales demandaron de los estudiantes bajo esfuerzo cognitivo para responder. Si se parte de la taxonomía de Bloom (Owen, 2006), las respuestas se ubicaron en los niveles de conocimiento y comprensión. Si la referencia es la nueva taxonomía de los objetivos educaciones de Marzano (Marzano y Kendall, 2008), las preguntas del profesor provocaron respuestas ubicadas en el área de conocimiento, en la categoría de información. Las contestaciones de los estudiantes se caracterizaron por contener en su totalidad una cita bibliográfica, o fueron escuetas, con poco nivel de argumentación y reflexión.

Igualmente, fue demostrado que cuando el profesor realizó planteamientos más elaborados y que representaban un dilema o una situación problemática, las respuestas de los estudiantes fueron más razonadas y eventualmente planteaban un ejemplo de la cotidianidad para justificar una postura; tal fue el caso de las respuestas obtenidas en la fase de Integración y de Exploración.

Los resultados indican que efectivamente hay una relación estrecha entre el tipo de mediación o facilitación, la propuesta de trabajo que se realiza dentro de los foros y los procesos sociocognitivos que se evidencian en los estudiantes. Esta conclusión es coincidente con la propuesta de Shea, Li y Pickett (2006), en cuanto a los elementos que son inherentes a la presencia docente y cómo impactan en el aprendizaje.

En esta misma línea, Garrison y Anderson afirman que, si no se evidencian la tercera y la cuarta fase del modelo bidimensional de investigación práctica (Integración y Resolución), se debe a la ausencia de una presencia docente que sea capaz de hacer "avanzar el discurso y desarrollo cognitivo individual a través de cada una de las fases de la investigación práctica" (2005, p. 93). De ahí la importancia de que el profesor, como mediador, sea capaz de diseñar y promover un ambiente de aprendizaje que logre resultados de alto nivel.
En cuanto al otro elemento inherente a la presencia docente, la propuesta de trabajo, se demostró en esta investigación que cuando el profesor acudió a estrategias que conllevaran a procesos de pensamiento de orden superior, como la presentación de un caso, los estudiantes buscaron argumentos dentro de la teoría y dentro del contexto cotidiano que les proveyeran las respuestas; por lo tanto, se manifestó un proceso más reflexivo y crítico. Tal como lo indican Garrison y Cleveland-Innes (2005), la presencia docente contribuye a la adopción de un enfoque profundo del aprendizaje, confirmando que la interacción por sí misma no es suficiente como elemento generador para garantizarlo. Esto es, hay una influencia del enfoque que permea el diseño y la enseñanza sobre los resultados de aprendizaje, ya sean estos profundos o superficiales.

\section{CONCLUSIONES}

Factores propios del actuar del profesor son determinantes en el desarrollo del pensamiento crítico en los estudiantes y evidenciado dentro de los foros de discusión en línea. Por esta razón, la planificación y el diseño de foros de discusión deben hacerse con mayor claridad en cuanto a la importancia de la mediación como tal y de las actividades y evaluación pertinentes a los objetivos que se proponen. En este último aspecto es vital reconocer cuál es el alcance que se pretende, si son niveles bajos de pensamiento o si se desea, sobre todo a nivel de posgrado, el análisis argumentado, la resolución de problemas, la toma de decisiones, todas ellas características del pensamiento crítico.

La presencia docente debe también estar dirigida a potenciar la metacognición, de manera tal que el desarrollo del pensamiento crítico no sea una responsabilidad absoluta del docente, sino que el estudiante tenga una conciencia clara de los procesos que le permiten conocer, ampliar y modificar la estructura cognitiva y llegar a nivel de pensamiento de orden 
superior. Lograr ampliar los procesos de la fase de Exploración a partir de la búsqueda de información para conocer y argumentar podría desembocar en mejores porcentajes en la fase de Resolución y, por lo tanto, en mayor incidencia de pensamiento reflexivo y crítico.

Se recomienda que futuras investigaciones analizar los efectos del tiempo que se tarda el profesor en proveer retroalimentación, en el estímulo y potenciación en los estudiantes del pensamiento crítico. Igualmente, se hace necesario ahondar en los roles que asumen los estudiantes dentro de un grupo y cómo éstos inciden en el desarrollo del pensamiento y la argumentación reflexiva. Aunado a este último aspecto será importante considerar la influencia que ejerce la empatía social y el compromiso que se deriva de ella, para llegar a niveles de intercambio de información en donde se busque la construcción colectiva de conocimiento. Se reconoce que el factor social es uno de los andamios que sostiene la reflexión y la argumentación crítica, pero no puede ni debe quedarse solo en la interacción.

\section{REFERENCIAS}

Anderson, T., Rourke, L., Garrison, D. \& Archer, W. (2001). Assessing teacher presence in a computer conferencing context. Journal of Asynchronous Learning Networks, 5(2). Recuperado el 3 de octubre del 2008, de http://www.aln.org/ publications/jaln/v5n2/pdf/v5n2_anderson.pdf

Bautista, G., Borges, F. \& Forés, A. (2008). Didáctica universitaria en entornos virtuales de enseñanza-aprendizaje (2a. ed.). Madrid, España: Narcea, S.A. Ediciones.

Bender, T. (2003). Discussion-based online teaching to enhance student learning. Theory, practice and assessment. Sterling, Virginia: Stylus Publishing.

Bruning, R., Schraw, G. \& Ronning, R. (2007). Psicología cognitiva e instrucción. Madrid, España: Alianza Editorial, S.A.
Cabero, J. (2000). La aplicación de las TIC: ¿esnobismo o necesidad educativa? Red digital: Revista de Tecnologías de la Información y Comunicación Educativas, (1). Recuperado el 3 de octubre del 2008, de http://reddigital.cnice.mec. es/1/firmas/firmas_cabero_ind.html

Cabero, J. \& Román, P. (2006). E-actividades. Un referente básico para la formación en Internet (1a. ed.). Sevilla, España: Editorial MAD, S.L.

Consejo de Rectoría (2007). Acuerdo 280-2007. Sesión No. 1486-2007, Art. V. San José, Costa Rica: Universidad Estatal a Distancia.

Crespo, A. (2006). Cognición Humana. Mente, ordenadores y neuronas (2a. ed.). Madrid, España: Editorial Universitaria Ramón Areces, S.A.

Duart, J. \& Sangrà, A. (2000). Aprender en la virtualidad (1a. ed.). Barcelona, España: Editorial Gedisa, S.A.

Ennis, R. (1993, Summer). Critical thinking assessment. Theory into Practice, 32(3), 179-186.

Gamboa, Y. \& Salas, I. (2009, Noviembre). Identificación de la percepción de los estudiantes de la Universidad Estatal a Distancia (UNED) sobre la usabilidad y los beneficios que obtienen de la plataforma WebCT al llevar a cabo sus procesos de aprendizaje. En Memoria X Encuentro Internacional Virtual Educa 2009, Buenos Aires, Argentina. Recuperado de: http://www.virtualeduca. info/ponencias2009/509/Ponencia\%20Yahaira\%20Gamboa-Ileana\%20Salas-set2009.pdf

García, B., Márquez, L., Bustos, A., Miranda, G. \& Espíndola, S. (2008). Análisis de los patrones de interacción y construcción del conocimiento en ambientes de aprendizaje en línea: una estrategia metodológica. Revista Electrónica de Investigación Educativa, 10(1). Recuperado de http://redie.uabc.mx/voliono1/contenidobustos.html\%3Ehttp://redie.uabc.mx/vol10no1/contenido-bustos.html

García, L., Ruiz, M. \& Domínguez, D. (2007). De la educación a distancia a la educación virtual (1a. ed.). Barcelona, España: Editorial Ariel, S.A.

Garrison, D. \& Anderson, T. (2005). El e-learning en el siglo XXI. Investigación y práctica (1a. ed.). Barcelona, España: Ediciones Octaedro, S.L. 
Garrison, D., Anderson, T. \& Archer, W. (2001). Critical thinking, cognitive presence, and computer conferencing in distance education. American Journal of Distance Education, 15(1), 7-23.

Garrison, D. \& Cleveland-Innes, M. (2005). Facilitating cognitive presence in online learning: Interaction is not enough. American Journal of Distance Education, 19(3), 133-148.

Gisbert, M. (2002). El nuevo rol del profesor en entornos tecnológicos. Acción Pedagógica, 11(1), 48-59.

Henning, P. (2004). Everyday cognition and situated learning. En: D. Jonassen (Ed), Handbook of research on educational communications and technology (pp.143-168). Mahwah, NJ: Lawrence Erbaum Associates.

Hernández, R., Fernández, C. \& Baptista, P. (2006). Metodología de la investigación (4a. ed.). México: McGraw-Hill Interamericana.

Marzano, R. \& Kendall, J. (2008). Designing \& assessing educational objectives: applying the new taxonomy. Thousand Oaks, California: Corvin Press.

Mayer, R. (1986). Pensamiento, resolución de problemas y cognición (1a. ed.). Barcelona, España: Ediciones Paidós Ibérica, S.A.

Melaré, D. (2007). Tecnologías de la inteligencia. Gestión de la competencia pedagógica virtual. España: Editorial Popular.

Monke, L. (2005, September-October). Charlotte's Web Page. Why children shouldn't have the world at their fingertips. Orion Magazine. Recuperado de http://www.orionmagazine.org/ index.php/articles/article/159/

Norris, S. \& Ennis, R. (1989). Evaluating critical thinking. The Practitioners' Guide to Teaching Thinking Series. Pacific Grove, CA.: Critical Thinking Press y Software.

Oh, S. \& Jonassen, D. (2007). Scaffolding online argumentation during problem solving. Journal of Computer Assisted Learning, 23(2), 95-110.
Owen, L. (2006). Beyond Bloom. A new version of the cognitive taxonomy. Recuperado el 21 de enero de 2011, de http://www.uwsp.edu/education/lwilson/curric/newtaxonomy.htm

Perkins, D. (1997). La escuela inteligente. Del adiestramiento de la memoria a la educación de la mente. Barcelona, España: Editorial Gedisa S.A.

Programa de Aprendizaje en Línea (2001). Oferta de cursos en Microcampus. San José, Costa Rica: Universidad Estatal a Distancia.

Programa de Aprendizaje en Línea (2007). Informe Anual 2007. San José, Costa Rica: Universidad Estatal a Distancia.

Programa de Aprendizaje en Línea (2009). Informe Anual 2009. San José, Costa Rica: Universidad Estatal a Distancia.

Salmon, G. (2002). Mirror, mirror on my screen. Exploring online reflections. British Journal of Educational Technology, 33(4), 379-391.

Salmon, G. (2003). E-moderating. The key to teaching and learning online. London, United Kingdom: Kogan Page Limited.

Salmon, G. (2004). E-actividades. El factor clave para una formación en línea activa. Barcelona, España: Editorial UOC.

Shea, P., Li, C. \& Pickett, A. (2006). A study of teaching presence and student sense of learning community in fully online and web-enhanced college courses. The Internet and Higher Education, 9(3), 175-190.

Tirado, R. (2002). Los entornos virtuales de aprendizaje. Bases para una didáctica del conocimiento. España: Grupo Editorial Universitario.

UNED. (2004). Modelo pedagógico de la Universidad Estatal a Distancia (1a. ed.). San José, Costa Rica: Vicerrectoría Académica.

UNED. (2008). Plan académico 2008-2011. San José, Costa Rica: Vicerrectoría Académica. 